Article

\title{
Genetic interaction between Mfrp and Adipor1 mutations affect retinal disease phenotypes.
}

\author{
Navdeep Gogna ${ }^{1 \sharp}$, Sonia Weatherly ${ }^{1 \sharp}$, Fuxin Zhao ${ }^{1,2}$, Gayle B Collin ${ }^{1}$, Jai Pinkney ${ }^{1}$, Lisa Stone ${ }^{1}$, Jurgen K Naggert ${ }^{1}$, \\ Gregory W Carter ${ }^{1}$ and Patsy M Nishina ${ }^{1, *}$
}

The Jackson Laboratory, 600 Main Street, Bar Harbor ME 04609, USA

2 School of Optometry and Ophthalmology and Eye Hospital, Wenzhou Medical University, Wenzhou 325027, Zhejiang, China.

\# Equal contributors

* Correspondence: patsy.nishina@jax.org; Tel.: +1-207-288-6383

\begin{abstract}
Adipor $1^{\text {tmIDgen }}$ and Mfrprde mutant mice share similar eye disease characteristics. Previously, studies established a functional relationship of ADIPOR1 and MFRP proteins in maintaining retinal lipidome homeostasis and visual function. However, the independent and/or interactive contribution of both the genes to similar disease phenotypes, including fundus spots, decreased axial length and photoreceptor degeneration has yet to be examined. We performed a gene-interaction study where homozygous Adipor $1^{\text {tm } 1 D g e n}$ and Mfrp ${ }^{r d 6}$ mice were bred together and the resulting doubly heterozygous F1 offspring were intercrossed to produce 210 F2 progeny. Four-month-old mice from all nine genotypic combinations obtained in the F2 generation were assessed for white spots by fundus photo documentation, for axial length by caliper measurements, and for photoreceptor degeneration by histology. Two-way factorial ANOVA was performed to study individual as well as gene interaction effects on each phenotype. Here, we report the first observation of reduced axial length in Adipor $1^{\text {tmlDgen }}$ homozygotes. We show that while Adipor1 and Mfrp interact to affect spotting and degeneration, they act independently to control axial length, highlighting the complex functional association between these two genes. Further examination of the molecular basis of this interaction may help in uncovering mechanisms by which these genes perturb ocular homeostasis.
\end{abstract}

Keywords: Mfrp; Adipor1; genetic interaction; fundus spots; photoreceptor degeneration; axial length.

\section{Introduction}

Membrane-type frizzled-related protein (MFRP), a transmembrane protein with two cubilin (CUB), a low-density lipoprotein receptor a (LDLRA), and a cysteine-rich (CRD)[1] domain, is expressed and localized in the retinal pigment epithelium (RPE) and ciliary body epithelium[2,3] of the eye. Disruptions in MFRP are known to cause a spectrum of human ocular diseases, including hyperopia[4], nanophthalmos[5,6], posterior microphthalmos[7], retinitis pigmentosa [8], foveoschisis[9,10] and optic disc drusen[11]. Likewise, mouse models with mutations in Mfrp, including $r d 6$ (4 bp deletion)[2], $r d x$ (174delG mutation)[12], and a c.498_499insC knock-in [13], show phenotypic similarities to the human diseases such as hyperopia, reduced axial length, retinal degeneration, RPE atrophy, and decreased electrophysiological response. The Mfrp mouse models also show the presence of uniformly sized and evenly distributed white spots across the fundus, similar to the white flecks observed in the patients with MFRP mutations[14,15] and in the human flecked retinal disorder retinitis punctata albescens[2,16].

In mouse, mutations in another gene, adiponectin receptor 1 (Adipor1), which encodes an integral membrane protein that localizes to RPE and photoreceptor (PR) cells[17], show 
disease characteristics similar to those found in Mfrp mouse models[12,13,17-19]. Mutations in Adipor1 also result in fundus spots and PR cell death[17]. While Mfrp is known to affect ocular growth[4,20,21], the same has not been studied in Adipor1 mutants. Mutations in ADIPOR1 have been associated with retinitis pigmentosa[18,19] and age-related macular degeneration[22]. While studies suggest that both ADIPOR1 and MFRP participate in docosahexaenoic acid (DHA, 22:6) enrichment[23] and lipid homeostasis in RPE and PR cells[24], there is still an incomplete understanding about the function of the individual proteins and the potential functional association and interaction between MFRP and ADIPOR1 that lead to similar disease phenotypes.

Quantitative genetic interaction/epistasis studies are a useful approach to examine functional relationships between genes and pathways[25-27]. Epistatic studies aim to determine the extent to which a particular phenotype in a double gene mutant varies from the phenotype observed in individual, single gene mutants[28]. However, such interaction studies are faced with complex interpretations when gene variants affect multiple phenotypes (pleiotrophy) and biological processes. Epistasis found for one phenotype may not be observed for another phenotype, making the interpretation about genetic interaction between two genes more complicated. As a first step towards unraveling the functional association between Adipor 1 and Mfrp, our study aimed to determine whether any of the ocular phenotypes were attributable to epistastic effects between mutations in these genes. Here, we performed a phenotypic assessment of Adipor $1^{\text {tm1Dgen }}$ and Mfrprd mice and their double mutant F2 progeny for fundus spotting, axial length (AL) and photoreceptor outer nuclear layer thickness. Using statistical approaches, we show that disruption in either gene contributes significantly to all the phenotypes assessed. Further, gene interaction modeling suggests that both mutant alleles interact to influence the severity of fundus spotting and PR cell degeneration, with no interaction effects on AL. Our study highlights the complexity of interactions between deleterious alleles of Adipor1 and Mfrp, which individually show very similar disease phenotypes but interact epistatically for some of them and act in isolation for other phenotypes.

\section{Results}

\subsection{Phenotypic similarities between $M$ frp ${ }^{r d 6}$ and Adipor ${ }^{\text {tm1Dgen }}$ mice}

Both Mfrprd6 and Adiportm1Dgen homozygotes present with small, uniformly sized white spots distributed pan-retinally (4-month-old depicted in Figure 1A). Outer nuclear layer thickness decreases relative to controls as photoreceptors undergo cell death (Figure 1B). Pigmented cells are found ectopically displaced in the subretinal space, consistent with the white spots observed by fundoscopy. A longitudinal study of the rate of PR degeneration in homozygous Adiportm1Dgen or Mfrprd6 mutants obtained from a (Adipor ${ }^{\text {tm1Dgen }} \mathrm{X}$ Mfrprd6) F2 cross indicates that the PR degeneration in homozygous Adipor $1^{\text {tm1Dgen }}$ mutants occur at a slightly faster rate than in Mfrprd6 mutant mice of the same age (Figure 1C).

Axial length, a key determinant of the refractive state of the eye, was also examined. A reduction in AL was observed in single homozygotes relative to controls by 10 weeks of age and progressed, such that by 4 months of age it was significantly different for both single mutants when compared to WT controls (Figure 1D). Refractive errors occur when light cannot focus properly on the retina, due to the altered shape of the eye. It can be caused by changes in axial length, or shape of the cornea or lens. Since AL is suggested to be a major contributor of refractive error[29] and we observed a change in AL for both the mutants, we also assessed refractive error. Our measurement of refractive error using an infrared photorefractor also confirmed significant changes ( $\mathrm{p}$-value $<0.0001$ ) in refraction at 10 weeks of age (Supplementary Figure S1), when the refractive errors of both Adipor1 ${ }^{t m 1 D g e n}$ and Mfrprd6 homozygotes shifted towards hyperopia. 


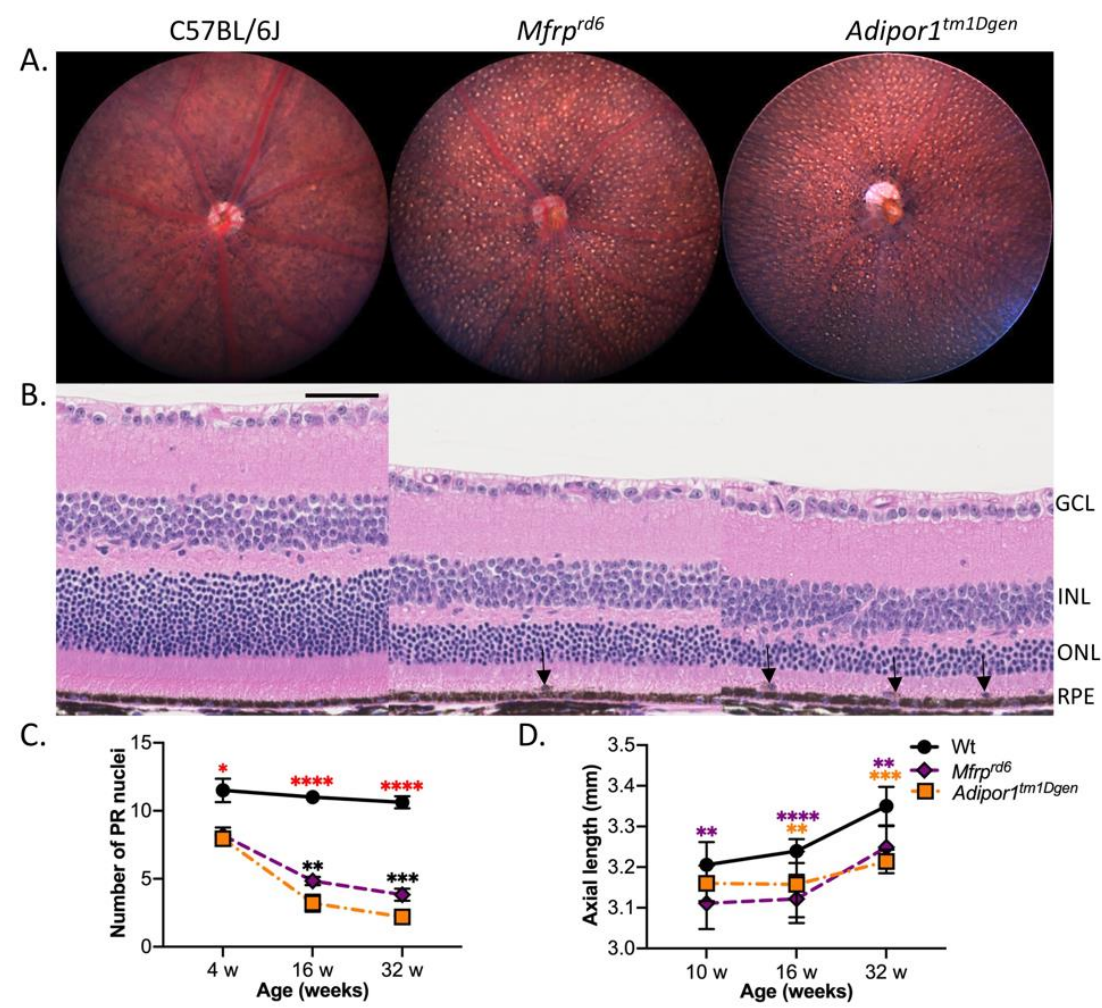

Figure 1. Mfrp ${ }^{\text {rd6 }}$ and Adipor $1^{\text {tm1Dgen }}$ mutant mice have similar ocular phenotypes. (A) Fundus photographs show evenly distributed discrete white spots throughout the retina of 4-month-old homozygous Mfrp ${ }^{r d 6}$ and Adipor ${ }^{\text {tm1Dgen }}$ mice, respectively, as compared to age-matched C57BL/6J controls. $\mathrm{N}=9-12$ per strain; both sexes included. (B) Retinal sections stained with hematoxylin and eosin (H\&E) and visualized by light microscopy reveal significant photoreceptor degeneration by 4 months of age in both mutants compared to age-matched C57BL/6J controls. Aberrant nucleated cells containing melanin pigment, as described by Hawes et al.[16], are observed in the subretinal space (black arrows) of mutant retinas only. $\mathrm{N}=10$ for each strain. Scale bar $=50$ microns. Longitudinal quantification of (C) photoreceptor degeneration (\#PR nuclei/300 $\mu \mathrm{m}$ length) and (D) axial length changes in single homozygotes from (Adipor ${ }^{\text {tm1Dgen }} \times \mathrm{M}_{\mathrm{fr}}{ }^{r d 6}$ ) F2 progeny: orange, homozygous for Adipor $1^{\text {tm1Dgen }}$ and wt for Mfrp ${ }^{\text {rd6; }}$; purple, wt for Adipor $1^{\text {tm1Dgen }}$ and homozygous for Mfr ${ }^{\text {rd6 }}$; and black, wt for both Adipor1 $1^{\text {tm } 1 D g e n}$ and Mfrp ${ }^{r d 6}$. Asterisks $\left(^{*}\right)$ represent the level of significance (ANOVA) between mutant and control mice; red asterisks indicate that both strains shared the same degree of significance with respect to the control, orange asterisks indicate significance between wt and Adipor $1^{\text {tm1Dgen }}$, purple asterisks indicate significance between wt and Mfr $p^{r d 6}$ and black asterisks indicate significance between Adipor $1^{\text {tm1Dgen }}$ and Mfr $p^{r d 6}$. P $>0.01\left(^{* *}\right), \mathrm{P}>0.0001\left(^{* * *}\right)$. For PR degeneration, $\mathrm{N}=4-6$; for axial length, $\mathrm{N}=8-15$ mice. Both sexes combined.

\subsection{Effects of Adipor1 ${ }^{\text {tm1Dgen }}$ and/or Mfrp ${ }^{\text {rd }}$ alleles on fundus appearance}

The similarity in Adipor $1^{\text {tm } 1 D g e n}$ and $M$ frr ${ }^{r d 6}$ fundus spotting phenotypes raised the possibility that these genes might interact in the same disease process. To test for such an interaction, we intercrossed the two mutants and examined the fundus phenotype at four months of age (Figure 2). In F2 progeny, results of funduscopic examination are shown in Figure 2A with the presence of panretinal white spots in singly homozygous Adipor1tm1Dgen and Mfrprd6 as well as doubly homozygous (Adipor1/Mfrp) mutants. Fundus spots were also seen in heterozygous Adipor $1^{+-}$and double heterozygous Adipor $1^{+-/} \mathrm{Mfrp}^{+/ r d 6}$ mice but not in heterozygous $M \mathrm{fr}^{+1 / \mathrm{rd} 6}$ mice. To determine if the spotting frequency among the genotypically unique cohorts significantly differed from that of wild type mice, all nine genotypes of F2 mice were evaluated in a masked fashion (central fundus images, estimated to encompass $\sim 1 / 6$ th of the entire eye, were obtained and identified by code numbers, randomized, and evaluated without knowledge of the genotype at the time of scoring) on an ordinal scale of $0-1$, where less than or equal to five spots was represented as 0 and the presence of more than five uniformly sized spots was represented as 1. 
To examine the effect of individual gene mutations and their interaction to affect fundus spots, a full factorial approach for the design of the experiment with a standard least squares model was used to construct a linear model in JMP statistical analysis software, through the fit model function. The model for spots phenotype had an adjusted $\mathrm{R}^{2}$ value of 0.7056 , root mean square error (RMSE) value of 0.2160 and a significant $F$ Ratio of 19.2792 (Prob $>\mathrm{F}=<0.0001$ ). The effects test confirmed the individual gene effect as well as gene interaction effect on the fundus spots phenotype (Supplementary Table S1). The results were also validated by statistical modeling using the R package, where the additive and interaction models were generated and compared to find which model out of the two was a better fit for the fundus spots data. The comparison between additive and interactive modelling confirmed the interaction model to be a better fit than the purely additive model as shown in Figure $2 \mathrm{~B}$ and $2 \mathrm{C}$ (adjusted $\mathrm{R}^{2}$ value for additive vs. interacting models: 0.5169 vs. 0.7056 ). An F-ratio test of the F-statistic derived from the two statistical models was significant, indicating superior performance of the interacting model (F-statistic and $p$ values: 10.9 and $p=1.2 \times 10^{-6}$, respectively). Given that heterozygous Adipor $1^{+/}$mice exhibit spots, statistical analysis also confirmed a significant effect of the heterozygous genotype for Adipor1 (Supplementary Table S2). However, the spots appear earlier and in greater numbers in the double heterozygous mice than in heterozygous Adipor $1^{+/}$alone (by approximately 10 weeks of age, many spots were seen in double heterozygous mice whereas very few to none were observed in heterozygous Adipor $1^{+/}$mice at the same age. Representative image shown in Supplementary Figure S2), suggesting an epistatic interaction effect between Adipor1 and Mfrp gene variants for the severity of fundus spots. For the $M f r p^{r d 6}$ allele, no differences were observed between wild type and heterozygous Mfrp ${ }^{+r d 6}$ mice, whereas homozygous mutants showed fundus spots, confirming the recessive mode of inheritance of the Mfrp rd allele for the spotting phenotype.

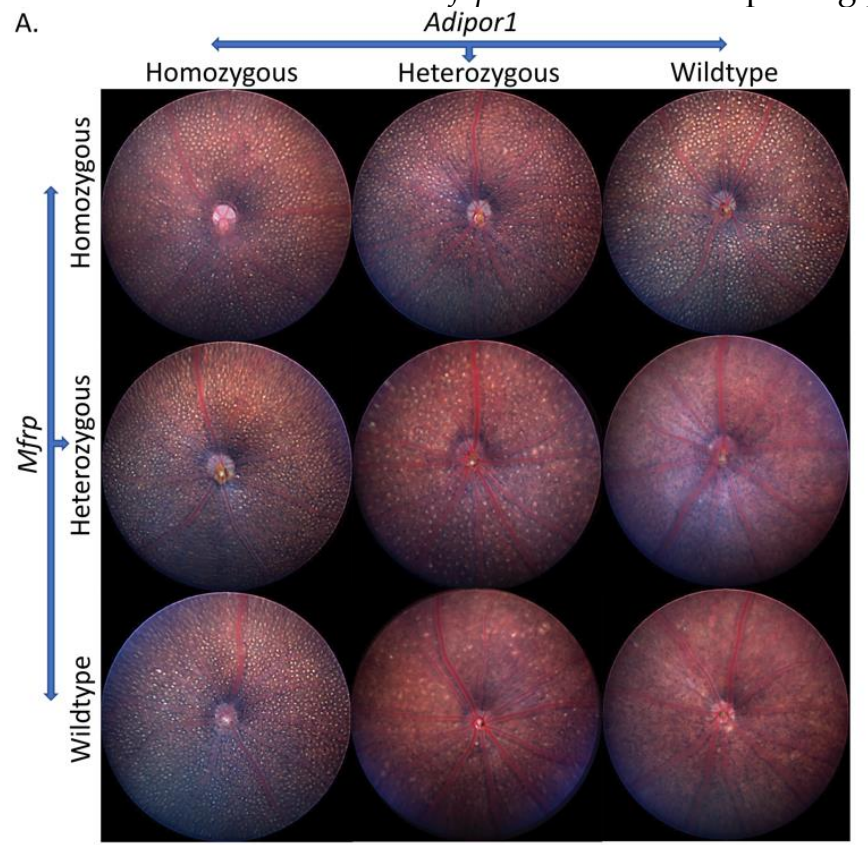

B.

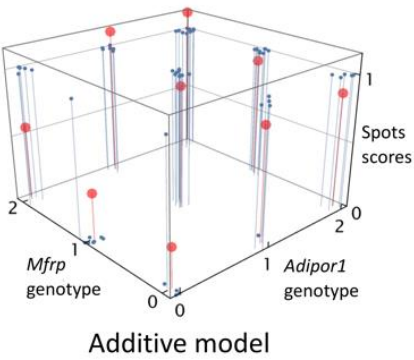

C.

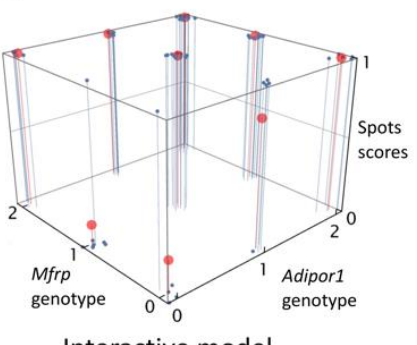

Interactive model

Figure 2. Fundus spots in 4-month-old F2 progeny, obtained from (Adipor $1^{+1-/ M f r p^{+/ r d 6}}$ ) F1 intercross. (A) Fundus photographs for mutants bearing one of the nine possible combinations of mutations, showing the presence of fundus spots in single and double homozygous mutants as well as double heterozygous mutants. Spots were also observed in heterozygous Adipor $1^{+/-}$mice. (B) Linear fit model without interactions and (C) Linear fit model with interactions, showing actual data in blue and model predictions in red. The Adipor1 and Mfrp genotypes along the two axis represent independent variables and the alleles are scored as: wildtype -0 , heterozygous -1 and homozygous -2 . The scores for fundus spots along the third axis represent response variables and are scored as 0 or 1 . Model predictions have a better fit with the actual data in the interactive model as compared to the additive model, confirming an interactive effect. $\mathrm{N}=5-10$ per genotype. 


\subsection{Effects of Adipor1 ${ }^{\text {tm1Dgen }}$ and/or Mfrp ${ }^{\text {rd6 }}$ alleles on axial length (AL)}

To study the effect of individual gene mutations as well as their interaction on AL changes, the full factorial approach, similar to the one used for fundus spot analysis, was used for the design of the experiment, and a standard least squares approach was used to construct a linear model in JMP software, to statistically analyze the ALs of all nine possible genotypes. The interaction model obtained for AL had an adjusted $\mathrm{R}^{2}$ value of 0.5160 , RMSE value of 0.0461 and a significant $F$ Ratio value of 24.9958 (Prob $>F=<0.0001$ ). The effects tests confirmed individual gene contributions to the axial length (Supplementary Table S3). However, the gene interaction effect was not significant (Prob $>F=0.5715)$. Tukey's HSD posthoc analysis revealed significant changes among nine double gene genotypes as shown in Figure 3A. The parameter estimates obtained using statistical analysis (Supplementary Table S4) showed no significant effect of the zygosity in case of either Adipor1 or Mfrp mutant alleles, confirming the recessive nature of inheritance for decreased axial length for both genes. The results were also validated by statistical modeling using the $\mathrm{R}$ package where the gene interaction model was not a better fit than the purely additive model as shown in Figure $3 \mathrm{~B}$ and $3 \mathrm{C}$ (adjusted $\mathrm{R}^{2}$ value for additive vs. interacting models: 0.519 vs. 0.516). An F-ratio test of the F-statistic derived from the two statistical models was not significant, indicating that the interacting model was not a significantly better fit than the additive model and there was no epistasis for the axial length phenotype (F-statistic and $p$ values: 43.7 and $p=0.56$, respectively).

A.

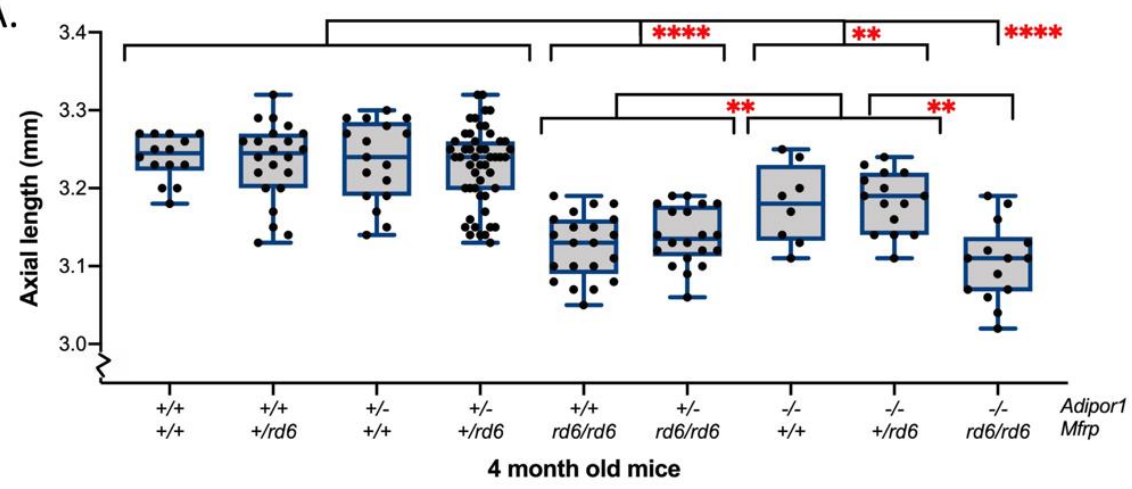

B.
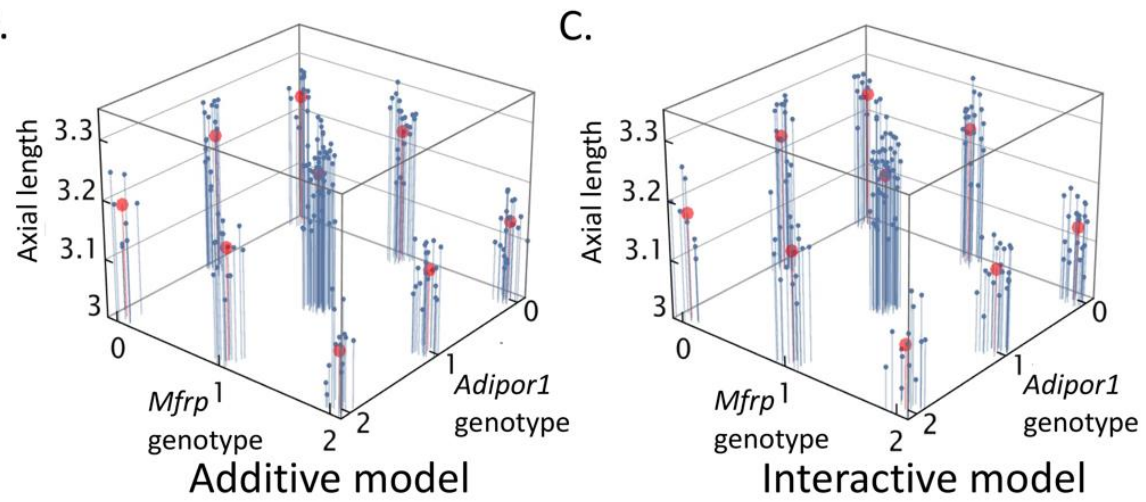

Figure 3. Axial length in 4-month-old F2 progeny obtained from an (Adipor $\left.1^{+1-/ M f r p+r d 6}\right)$ ) F1 intercross. (A) Using two-way factorial-ANOVA, followed by Tukey's HSD posthoc analysis revealed statistically significant differences in axial length between different genotypic possibilities for the F2 intercross progeny. As no gender-based differences were found, data from both genders were combined. Single asterisk $\left({ }^{*}\right)$ indicates $\mathrm{p}<0.05$; two asterisks $\left(^{* *}\right)$ indicate $\mathrm{p}<0.01$; three asterisks $\left({ }^{* * *}\right)$ indicate $\mathrm{p}<0.001$ and four asterisks $\left(^{* * * *}\right)$ indicate $\mathrm{p}<0.0001$. (B) Linear fit model without interactions and (C) Linear fit model with interactions, showing actual data in blue and model predictions in red. The Adipor 1 and Mfrp genotypes along the two axis represent independent variables, where the alleles are scored as: wildtype -0 , heterozygous -1 and homozygous -2 . The axial length measurements along the third axis represent response variables. The interactive model does not 
have a better fit than the additive model, confirming no interaction and independent effects. $\mathrm{N}=14$ 50 per genotype.

\subsection{Effects of Adipor ${ }^{\text {tm1Dgen }}$ and/or Mfrp $^{\text {rd6 }}$ alleles on Photoreceptor (PR) degeneration} To examine the effect of individual genes as well as their interaction on PR degeneration, a linear model was again constructed using a full factorial design of the experiment with a standard least squares model, similar to the approach used for fundus spot and AL analysis. For PR nuclear count, hematoxylin and eosin-stained sections were used for measurements. Starting at the optic nerve $(\mathrm{ON})$, the sections were divided into 300 micron long intervals along the length of the retina, on both sides of the ON. Excluding the first 300 micron interval, the nuclei within the next 300 micron region of interest (ROI) were counted, and values were reported using a semi-automated approach as described in materials and methods. Final nuclei count values were reported as average nuclei counts within a 300 micron length on either side of the ON. For PR nuclear counts, an adjusted $\mathrm{R}^{2}$ value of 0.9384 , root mean square error (RMSE) of 0.1878 and a significant $F$ Ratio of 216.4440 (Prob $>\mathrm{F}=<0.0001$ ) resulted from the interaction model revealing a significant main effect of both genes as well as a significant gene interaction effect on PR degeneration as shown in the effect tests table obtained from the JMP analysis (Supplementary Table S5). Tukey's HSD post-hoc test was used to confirm the statistically significant differences in the PR degeneration between the nine genotypic possibilities for the Adipor1/Mfrp mutants as shown in Figure 4A. The parameter estimates obtained using statistical analysis (Supplementary Table S6) show significant effect of the zygosity in case of Adipor1, which suggests a semi-dominant nature of inheritance. The results were validated from the statistical modeling using the $\mathrm{R}$ package where the interaction and additive models were compared, and confirmed that the gene interaction model was a better fit than the purely additive model as shown in Figure $4 \mathrm{~B}$ and $4 \mathrm{C}$ (adjusted $\mathrm{R}^{2}$ value for additive vs. interacting models: 0.846 vs. 0.938). An F-ratio test of the F-statistic derived from the two statistical models was significant, indicating superior performance of the interacting model (Fstatistic: 43.7 and $\mathrm{p}=7.5 \times 10-22)$.
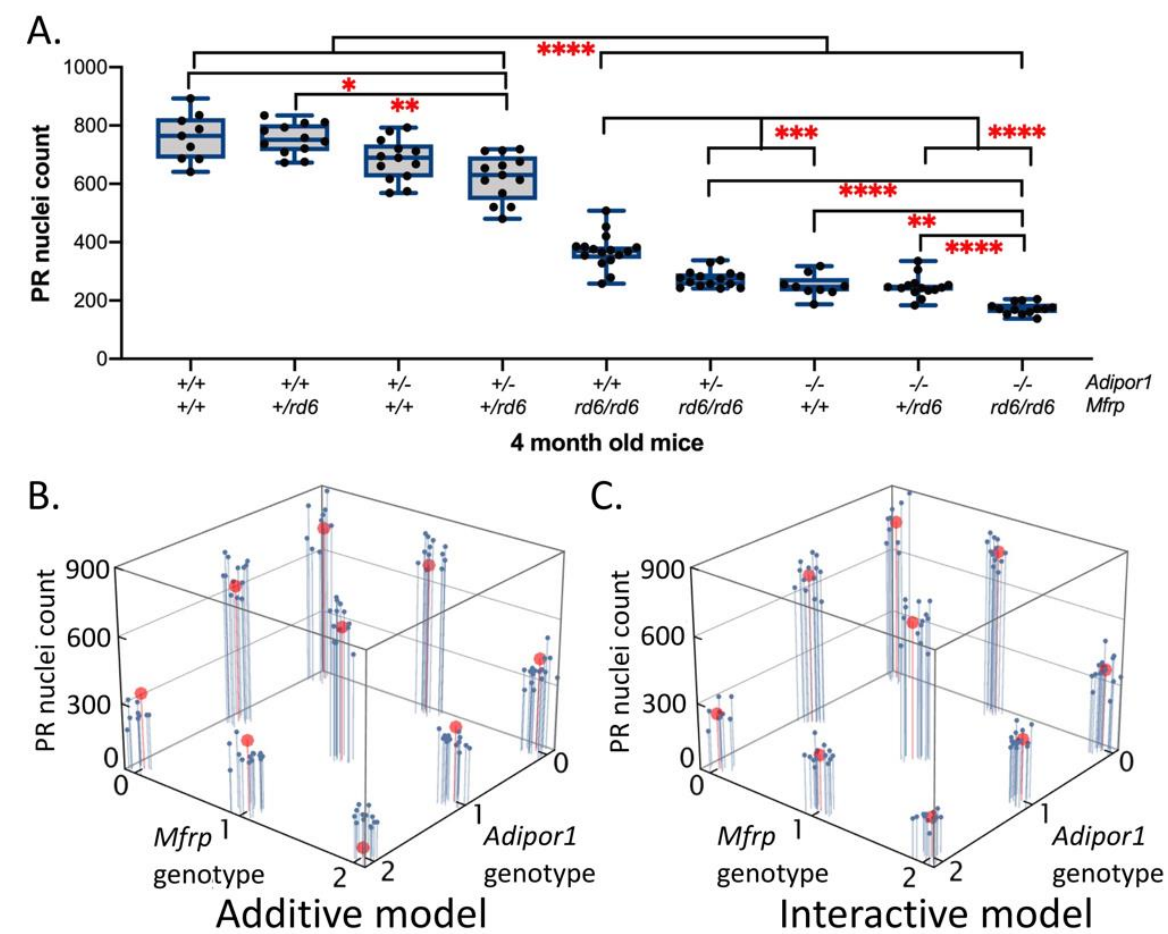

Figure 4. Photoreceptor degeneration in 4-month-old F2 mice from an (Adipor1 $1^{\left.+\downarrow-/ M f r p^{+/ r d 6}\right)}$ F1 intercross: (A) Using two-way factorial-ANOVA, followed by Tukey's HSD posthoc analysis revealed statistically significant differences in PR degeneration between different genotypic possibilities of F2 intercross progeny. Absence of additive effect in single (Adipor $1^{-/ /} / \mathrm{Mfr}^{+/+}$, Adipor $1^{+/+} / \mathrm{Mfrp}^{r d 6 / r d 6}$ ) 
and double (Adipor ${ }^{-1} / M$ frp ${ }^{r d 6 / r d 6}$ ) gene homozygous mutants, as compared to the wild types (Adi$\left.\operatorname{por}^{+++} / \mathrm{Mfr}^{+/+}\right)$, confirmed the gene interaction effects. As no gender-based differences were found, data from both genders were combined. Single asterisk $\left(^{*}\right)$ indicates $p<0.05$; two asterisks $\left({ }^{* *}\right)$ indicate $\mathrm{p}<0.01$; three asterisks $\left({ }^{* * *}\right)$ indicate $\mathrm{p}<0.001$ and four asterisks $\left({ }^{* * * *}\right)$ indicate $\mathrm{p}<0.0001$, (B) Linear fit model without interactions and (C) linear fit model with interactions, showing actual data in blue and model predictions in red. The Adipor 1 and Mfrp genotypes along the two axis represent independent variables, where the alleles are scored as: wildtype - 0 , heterozygous -1 and homozygous -2 . The PR nuclei count along the third axis represents response variables. The model predictions fit the actual data better in the interactive model, so it's concluded that the interactive model has a better fit than the additive model, confirming an interactive effect. $\mathrm{N}=9-15$ per genotype.

\section{Discussion}

Although both Adipor $1^{\text {tm1Dgen }}$ and Mfrp ${ }^{\text {rd }}$ mutant mice show very similar retinal disease characteristics, there is an incomplete understanding about the functional relationship between these two genes. Particularly, the individual contributions of Adipor 1 and Mfrp and their functional association to regulate phenotypes including fundus spots, AL changes, and PR degeneration has not been examined. Studies involving epistatic interactions, where the phenotypic impact of one gene depends on another gene, have been routinely used to expose functional associations[30,31]. We designed our study on the same principle, where the information obtained from epistatic interaction(s), inferred from the phenotypic measurements, was used to elucidate the potential functional association between Adipor $1^{\text {tm } 1 D g e n}$ and Mfrprd6. We performed genetic interaction studies by comparing quantitative phenotypes of double mutant Adipor $1^{\text {tm1Dgen }} / \mathrm{M}$ frp rd allelic combinations to single mutant (Adipor ${ }^{\text {tm1Dgen }}$ and $M$ frp ${ }^{r d 6}$ ) alleles, respectively. We measured three retinal phenotypes known to be affected in both Adipor $1^{\text {tm1Dgen }}$ and $M$ frp ${ }^{r d 6}$ single gene mutants.

Both Adipor $1^{\text {tmIDgen }}$ and Mfrprd6 homozygous mutants present with very similar spots by fundus photography. The white spots, for both mutants, correspond to the sub-retinally accumulated macrophages/monocytes, identified by immunolabelling with macrophagespecific markers MOMA-2 and F4/80+[12,16,17,32]. This suggested the possibility that the genes may be functionally associated and follow similar biological processes of initiating an immune response to develop fundus spots. Our results from the epigenetic analysis confirmed significant individual gene as well as gene interaction effects on the spots phenotype. This shows that in addition to independent effects, perhaps through mutant-specific induced pathways, the fundus spotting due to loss of ADIPOR1 is also dependent on the fundus spotting due to loss of MFRP and vice versa, suggesting that some mechanistic aspects of the observed phenotype are also shared.

Similar to fundus spots, Adipor1 ${ }^{\text {tm1Dgen }}$ and Mfrprd6 homozygotes also show a significant decrease in PR cell count due to degeneration. Docosahexaenoic acid (DHA) is an important component of PR outer segment membranes and is vital for RPE and PR cell functions[33-35]. Studies have suggested that Adipor1 acts as a regulatory switch for the uptake, retention, conservation and elongation of DHA in PR and RPE cells, thus preserving PR cell integrity[17]. Recently, it has been suggested that Mfrp participates in DHA enrichment similar to Adipor1[24]. This suggests that these genes may be involved in regulating retinal lipidome homeostasis and that a decrease in DHA, very long chain-polyunsaturated fatty acids (VLC-PUFAs) and the inability to synthesize neuroprotective elovanoids along with the activation of inflammatory signaling pathways may lead to PR instability and degeneration[24,36] in both Adipor1 $1^{\text {tm } 1 D g e n}$ and Mfrprd6 homozygotes. Our results from the epigenetic analysis also confirmed significant individual gene as well as gene interaction effects on the PR degeneration. Our results demonstrate that both Adipor 1 and Mfrp are functionally associated for maintaining PR integrity, possibly through shared or different mechanisms that affect DHA enrichment. 
For the Adipor1 gene mutant, a significant effect of zygosity was observed for both fundus spots and PR degeneration phenotypes. While fundus spots are an expected phenotype in homozygous Adipor1 and Mfrp mutants, it was interesting that the double heterozygous mutants as well as mice heterozygous for the Adipor1 mutation (Adipor $1^{+/} / \mathrm{M} \mathrm{rrp}^{+++}$) alone also developed a spotting phenotype. Similarly, a decrease in PR cell count was also observed in heterozygous Adipor1 mice compared to age-matched controls. This confirmed that the presence of one wild-type allele of Adipor1 is not sufficient to prevent either of these phenotypes. This is in contrast with a previous study where no statistically significant differences were found in heterozygous and wild type Adipor1 mice for the visual system protein levels measured, indicating that $50 \%$ of ADIPOR1 levels was sufficient for retinal stability[37]. However, that study was performed at P15 and P22. It is possible that the effect of zygosity is more pronounced with aging as is the case of our 4-month-old animals. Additionally, the observation of more fundus spots, at an earlier age in double heterozygous mice than in heterozygous Adipor1 mice, suggests a possible interactive effect of Mfrp on the Adipor1 phenotype. Concomitant with the increase in fundus spots is a greater relative decrease in PR nuclei in mutants bearing Adipor1 loss-of-function alleles as compared to the mutants bearing loss of $M$ frp alleles (Figure 2 and 4). It is reasoned that since homozygous Mfrprd6 mice lack ADIPOR1 protein in their RPE layer[37], it is likely that the partial RPE ADIPOR1 deficiency contributes to the PR degeneration observed in $M f r r^{r d 6}$ homozygotes. However, since ADIPOR1 is retained in the retina of Mfrr ${ }^{r d 6}$ homozygotes, there is relatively less PR degeneration and fewer fundus spots in Mfrprd6 homozygotes as compared to the Adipor1 ${ }^{\text {tm } 1 D g e n}$ homozygotes which have a complete loss of ADIPOR1 in both retina as well RPE.

Mutations in MFRP have been associated with reduced axial length[4,5,38]. A similar decrease in axial length has also been reported in Zebrafish Mfrp mutants[39]. While initial studies with $r d 6$ and $r d x$ mouse models did not report ocular axial length abnormalities[13,16], later studies with Mfrp KI/KI mice[13] and $r d 6$ mice[4] demonstrated decreased ocular axial length. Since MFRP contains a cysteine-rich domain essential for Wnt binding and signaling, which has been implicated in vertebrate eye development[40], studies have suggested the possibility that MFRP might regulate axial length changes through Wnt signaling $[2,13,41]$. Axial length changes associated with Adipor1 mutations have not been reported prior to this study. Our study confirms that similar to Mfrprde mutants, Adipor $1^{\text {tm } 1 D g e n}$ mutants also exhibit decreased axial lengths. However, in our epistatic analysis of axial length in both the single and double mutants from the F2 cross, we did not observe gene interaction effects. Therefore, it appears the two genes affect axial length in an independent, mutant specific manner.

Our study underscores that although both Adipor 1 ${ }^{\text {tm1Dgen }}$ and Mfrprd6 mutants share similar eye disease characteristics, the extent to which the phenotypes are affected differs in each of the mutants. Further, it also highlights the complexity of functional associations and interactions between these two genes, where the genes interact to exacerbate some phenotypes while contributing individually to others, and the analytical importance of epistatic studies in unravelling such associations.

\section{Materials and Methods}

\subsection{Ethics Statement}

Care and handling of mice in this study conformed to the Association for Research in Vision and Ophthalmology Resolution on the Use of Animals in Ophthalmic and Vision Research. All protocols involving mice were approved by The Jackson Laboratory Institutional Animal Care and Use Committee (IACUC, AUS99089), in accordance with the "Guide for the Care and Use of Experimental Animals" established by the National Institutes of Health (Bethesda, Maryland). 


\subsection{Animals}

Mouse strains used in this study, B6.129P2-Adipor1 ${ }^{\text {tm1Dgen }} / \mathrm{Mmnc}$ (MMRRC, stock \# 011599UNC), B6.C3Ga- Mfrprd6/J (The Jackson Laboratory, stock \# 003684) and C57BL/6J (The Jackson Laboratory, stock \# 000664), herein referred to as Adipor $1^{-1}$, Mfrprd6/rd6, and wildtype (WT), respectively, when used as double mutants, were bred and maintained under standard conditions of 12:12 light-dark cycle in the Research Animal Facility at The Jackson Laboratory. Mice were provided with NIH31 (6\% fat chow) diet and $\mathrm{HCl}$-acidified water $(\mathrm{pH}$ 2.8-3.2) ad libitum and maintained in pressurized individual ventilation caging, which were regularly monitored to ensure a pathogen-free environment. Both Adipor $1^{-1-}$ and Mfrprd6 are maintained on the C57BL/6J strain background and confirmed to be free of the $C r b 1^{r d s}$ mutation.

To identify individual gene contributions and to ascertain the possibility of a digenic epistatic interaction between Adipor1 and Mfrp gene mutations to modulate disease phenotypes, Adipor ${ }^{1 m 1 D g e n}$ and Mfrp ${ }^{r d 6}$ homozygotes were bred together to generate F1 mice that were double heterozygotes (Adipor $1^{+/} / M f r p^{+/ r d 6}$ ). The resulting F1 offspring were then intercrossed to obtain an F2 generation to generate nine possible genotypic combinations (Supplementary Figure S3). All F2 mice were analyzed for the development of fundus spots, axial length changes and photoreceptor degeneration at 4 months of age.

\subsection{Genotyping}

Genomic DNA was isolated from either tail tips $(<2 \mathrm{~mm})$ or ear punches. DNA was extracted by incubating the tissue in $50 \mathrm{mM}$ sodium hydroxide solution at $95^{\circ} \mathrm{C}$ for $30-50$ minutes, followed by the addition of $1 \mathrm{M}$ Tris $\mathrm{HCl}$ neutralizing reagent buffer to a final concentration of $200 \mathrm{mM}$. The sample was briefly vortexed and spun at 3,600 rpm for 8 minutes at $4^{\circ} \mathrm{C}$. Two $\mu \mathrm{l}$ of the supernatant containing the genomic DNA was then used per $12 \mu \mathrm{l}$ PCR reaction reagents. For Adipor1, primers used were: NIH62-GS1, TCCACTGTGTCAGCTTCTCTGTTAC, NIH62-GS2, AGGCAGGGTAAGCTGATTAGCTATG, and NIH62-neo, GGGTGGGATTAGATAAATGCCTGCTCT (Protocol from MMRRC at UNC \#11599; www.med.unc.edu/mmrrc). Amplicons (254-bp for wildtype and 433-bp for mutant) were visualized with EZ-vision In-Gel Solution (VWR, Catalog N391-15MLDRP) after electrophoretic separation on a 1.5\% agarose gel. The Mfrr rd6 mutation assay was carried out by the JAX genotyping facility using the available protocols (HYPERLINK "https://www.jax.org/Protocol?stockNumber=003684\&protocolID=32162"https://www.jax.org/Protocol?stockNumber=003684\&protocolID=32162 and https://www.jax.org/Protocol?stockNumber=003684\&protocolID=32010). https://www.jax.org/Protocol?stockNumber=003684\&protocolID=32010).

\subsection{Fundus Imaging}

Fundus examination was performed as previously described [42], using a Micron IV fundus camera (Phoenix Research Laboratories, Pleasanton, CA), with the exception that $1 \%$ cyclopentolate or $1 \%$ atropine was used as the dilating agent, and mice were anesthetized with isoflurane (isoflurane vaporizer from Kent Scientific, USA) for the duration of imaging.

\subsection{Histological Analysis}

Mice were euthanized by carbon dioxide asphyxiation. The enucleated eyes were placed in ice-cold methanol:acetic acid:PBS (3:1:4) solution for overnight fixation at $4^{\circ} \mathrm{C}$. The fixed eyes were subsequently paraffin embedded, cut into $6 \mu \mathrm{m}$ sections, stained with Hematoxylin \& Eosin, and visualized by light microscopy. Histological images were captured using a Nanozoomer digital slide scanner (Hamamatsu, Japan). The Fiji imaging processing package was used to assess PR cell loss from scanned images [43]. A custom Fiji macro was first used to extract tif images of single sections from full-resolution NanoZoomer output files, which exceded Fiji memory capabilities. A separate macro was then created to draw rectangular regions of interest (ROIs) encompassing a $300 \mu \mathrm{m}$ length of 
retina and spaced at $0.3 \mathrm{~mm}$ intervals starting from the optic nerve head on either side. PR cell loss was determined from .tif images by counting PR cell nuclei within each ROI by both, using a macro as well as manually.

\subsection{Axial Length Measurement}

Enucleated eyes were assessed for axial length (anterior to posterior) using Vernier calipers. Three measurements were taken for each eye. Since the measurements were not found to differ statistically between right and left eyes and no gender-based differences were observed within each genotype (assessed by t-test), the measurements from both sexes were combined into one cohort per genotype to compare axial length measurements between different genotypes.

\subsection{Refractive Error Measurement}

The refractive state was measured in a darkened room with a custom-built automated eccentric infrared photo refractor calibrated according to a published procedure[44]. The data was recorded using software designed by Schaeffel et al.[44], and each measurement was repeated a minimum of three times. No statistically significant difference was observed between the right and left eyes (statistical significance determined by paired twotailed Student's t-test), thus, the data from both eyes of each animal was averaged.

\subsection{Statistical Analysis}

t-test and ANOVA were performed using GraphPad Prism version 8 (GraphPad Software, San Deigo, CA, USA) and JMP statistical analysis software (SAS Institute, NC, USA). Individual gene contributions and genetic interaction effects were analyzed by two-way (factorial) ANOVA. Genes were selected as two categorical variables (factors) and the effect of their interaction was tested on each phenotype, selected as continuous (response) variable. To determine which means were different, a post hoc Tukey's HSD (for equal variances) or Dunnett's T3 (for unequal variances) multiple comparison test was applied. The data were tested for normal distribution and equal variances and corrected for unequal sample sizes. Linear fit modelling was performed using R statistical software, version 3.4.3.

Supplementary Materials: The following are available online at www.mdpi.com/xxx/s1, Figure S1: Significant changes in refractive error (Diopter) observed in homozygous Adipor $1^{\text {tm } 1 \text { Dgen }}$ and Mfrp ${ }^{\text {rd } 6}$ mice, by 10 weeks of age, Figure S2: Spots observed by fundus photodocumentation in 10-weeksold double heterozygous and heterozygous Adipor1 ${ }^{+/-}$mice, Figure S3: Generation of Adipor1/Mfrp double mutant mice. Table S1: Effect tests (which explains the significance of independent variables as well as their interaction in the model) obtained from fit model analysis of fundal spots, confirming significant effect due to individual mutations as well as due to gene interaction, Table S2: Parameter estimates obtained from fit test analysis for fundal spots, showing which variables/predictor have significant influence on the response, Table S3: Effect tests obtained from fit model analysis for axial length, confirming the absence of interaction between Adipor1 and Mfrp for determining axial length, Table S4: Parameter estimates obtained from fit test analysis for axial length, Table S5: Effect Tests obtained from fit model analysis for PR degeneration, confirming significant effect due to individual mutations as well as due to gene interaction, Table S6: Parameter estimates obtained from fit test analysis for PR degeneration. Table S7: Data sheet: Data for fundal spots, Table S8: Data for axial length and Table S9: Data for PR degeneration used for statistical analysis.

Author Contributions: For research articles with several authors, a short paragraph specifying their individual contributions must be provided. The following statements should be used "Conceptualization, G.W.C., J.K.N. and P.M.N; investigation , S. W., G.B.C., J.P., $\quad$ L.S., $\quad$ and $\quad$ F.Z., validation, W., G.B.C., J.P; formal analysis, N.G., and G.W.C; resources, L.S.; data curation, G.B.C., writingoriginal draft preparation, N.G.; writing-review and editing, all authors.; visualization, N.G., G.W.C; supervision, P.M.N.; funding acquisition, P.M.N. All authors have read and agreed to the published version of the manuscript." 
Funding: This research was funded by the National Eye Institute (NEI) grant, EY011996. Core services at The Jackson Laboratory were supported by an Institutional grant CA34196.

Institutional Review Board Statement: All protocols involving mice were approved by The Jackson Laboratory Institutional Animal Care and Use Committee (IACUC, AUS99089), in accordance with the "Guide for the Care and Use of Experimental Animals" established by the National Institutes of Health (Bethesda, Maryland).

Data Availability Statement: The data presented in this study are available in the supplementary material.

Acknowledgments: The authors thank the JAX Scientific Research Services, including the Histological and Transgenic Genotyping Services for genotyping mice strains, Melissa Berry for assistance with nomenclature, Jim Peterson for designing the algorithm for PR nuclei counting and Mark P. Krebs for his useful discussions.

Conflicts of Interest: The authors declare no conflict of interest.

\section{References}

1. Won, J.; Smith, R.S.; Peachey, N.S.; Wu, J.; Hicks, W.L.; Naggert, J.K.; Nishina, P.M. Membrane frizzled-related protein is necessary for the normal development and maintenance of photoreceptor outer segments. Vis Neurosci 2008, 25, 563-574, doi:10.1017/S0952523808080723.

2. Kameya, S.; Hawes, N.L.; Chang, B.; Heckenlively, J.R.; Naggert, J.K.; Nishina, P.M. Mfrp, a gene encoding a frizzled related protein, is mutated in the mouse retinal degeneration 6. Hum Mol Genet 2002, 11, 1879-1886, doi:10.1093/hmg/11.16.1879.

3. Mandal, M.N.; Vasireddy, V.; Jablonski, M.M.; Wang, X.; Heckenlively, J.R.; Hughes, B.A.; Reddy, G.B.; Ayyagari, R. Spatial and temporal expression of MFRP and its interaction with CTRP5. Investigative ophthalmology $\mathcal{E}$ visual science 2006, 47, 55145521, doi:10.1167/iovs.06-0449.

4. Velez, G.; Tsang, S.H.; Tsai, Y.T.; Hsu, C.W.; Gore, A.; Abdelhakim, A.H.; Mahajan, M.; Silverman, R.H.; Sparrow, J.R.; Bassuk, A.G.; et al. Gene Therapy Restores Mfrp and Corrects Axial Eye Length. Sci Rep 2017, 7, 16151, doi:10.1038/s41598017-16275-8.

5. Sundin, O.H.; Leppert, G.S.; Silva, E.D.; Yang, J.M.; Dharmaraj, S.; Maumenee, I.H.; Santos, L.C.; Parsa, C.F.; Traboulsi, E.I.; Broman, K.W.; et al. Extreme hyperopia is the result of null mutations in MFRP, which encodes a Frizzled-related protein. Proc Natl Acad Sci U S A 2005, 102, 9553-9558, doi:10.1073/pnas.0501451102.

6. Prasov, L.; Guan, B.; Ullah, E.; Archer, S.M.; Ayres, B.M.; Besirli, C.G.; Wiinikka-Buesser, L.; Comer, G.M.; Del Monte, M.A.; Elner, S.G.; et al. Novel TMEM98, MFRP, PRSS56 variants in a large United States high hyperopia and nanophthalmos cohort. Sci Rep 2020, 10, 19986, doi:10.1038/s41598-020-76725-8.

7. Aldahmesh, M.A.; Nowilaty, S.R.; Alzahrani, F.; Al-Ebdi, L.; Mohamed, J.Y.; Rajab, M.; Khan, A.O.; Alkuraya, F.S. Posterior microphthalmos as a genetically heterogeneous condition that can be allelic to nanophthalmos. Arch Ophthalmol 2011, 129, 805-807, doi:10.1001/archophthalmol.2011.129.

8. Dinculescu, A.; Estreicher, J.; Zenteno, J.C.; Aleman, T.S.; Schwartz, S.B.; Huang, W.C.; Roman, A.J.; Sumaroka, A.; Li, Q.; Deng, W.T.; et al. Gene therapy for retinitis pigmentosa caused by MFRP mutations: human phenotype and preliminary proof of concept. Hum Gene Ther 2012, 23, 367-376, doi:10.1089/hum.2011.169.

9. Godinho, G.; Madeira, C.; Grangeia, A.; Neves-Cardoso, P.; Santos-Silva, R.; Brandao, E.; Carneiro, A.; Falcao-Reis, F.; Estrela-Silva, S. A novel MFRP gene variant in a family with posterior microphthalmos, retinitis pigmentosa, foveoschisis, and foveal hypoplasia. Ophthalmic Genet 2020, 41, 474-479, doi:10.1080/13816810.2020.1795888.

10. Crespi, J.; Buil, J.A.; Bassaganyas, F.; Vela-Segarra, J.I.; Diaz-Cascajosa, J.; Ayala-Ramirez, R.; Zenteno, J.C. A novel mutation confirms MFRP as the gene causing the syndrome of nanophthalmos-renititis pigmentosa-foveoschisis-optic disk drusen. Am J Ophthalmol 2008, 146, 323-328, doi:10.1016/j.ajo.2008.04.029. 
11. Ayala-Ramirez, R.; Graue-Wiechers, F.; Robredo, V.; Amato-Almanza, M.; Horta-Diez, I.; Zenteno, J.C. A new autosomal recessive syndrome consisting of posterior microphthalmos, retinitis pigmentosa, foveoschisis, and optic disc drusen is caused by a MFRP gene mutation. Mol Vis 2006, 12, 1483-1489.

12. Fogerty, J.; Besharse, J.C. 174delG mutation in mouse MFRP causes photoreceptor degeneration and RPE atrophy. Investigative ophthalmology \& visual science 2011, 52, 7256-7266, doi:10.1167/iovs.11-8112.

13. Chekuri, A.; Sahu, B.; Chavali, V.R.M.; Voronchikhina, M.; Soto-Hermida, A.; Suk, J.J.; Alapati, A.N.; Bartsch, D.U.; AyalaRamirez, R.; Zenteno, J.C.; et al. Long-Term Effects of Gene Therapy in a Novel Mouse Model of Human MFRP-Associated Retinopathy. Hum Gene Ther 2019, 30, 632-650, doi:10.1089/hum.2018.192.

14. Neri, A.; Leaci, R.; Zenteno, J.C.; Casubolo, C.; Delfini, E.; Macaluso, C. Membrane frizzled-related protein gene-related ophthalmological syndrome: 30-month follow-up of a sporadic case and review of genotype-phenotype correlation in the literature. Mol Vis 2012, 18, 2623-2632.

15. Zenteno, J.C.; Buentello-Volante, B.; Quiroz-Gonzalez, M.A.; Quiroz-Reyes, M.A. Compound heterozygosity for a novel and a recurrent MFRP gene mutation in a family with the nanophthalmos-retinitis pigmentosa complex. Mol Vis 2009, 15, 17941798.

16. Hawes, N.L.; Chang, B.; Hageman, G.S.; Nusinowitz, S.; Nishina, P.M.; Schneider, B.S.; Smith, R.S.; Roderick, T.H.; Davisson, M.T.; Heckenlively, J.R. Retinal degeneration 6 (rd6): a new mouse model for human retinitis punctata albescens. Investigative ophthalmology \& visual science 2000, 41, 3149-3157.

17. Rice, D.S.; Calandria, J.M.; Gordon, W.C.; Jun, B.; Zhou, Y.; Gelfman, C.M.; Li, S.; Jin, M.; Knott, E.J.; Chang, B.; et al. Adiponectin receptor 1 conserves docosahexaenoic acid and promotes photoreceptor cell survival. Nat Commun 2015, 6, 6228, doi:10.1038/ncomms7228.

18. Zhang, J.; Wang, C.; Shen, Y.; Chen, N.; Wang, L.; Liang, L.; Guo, T.; Yin, X.; Ma, Z.; Zhang, B.; et al. A mutation in ADIPOR1 causes nonsyndromic autosomal dominant retinitis pigmentosa. Hum Genet 2016, 135, 1375-1387, doi:10.1007/s00439-016$1730-2$.

19. Xu, M.; Eblimit, A.; Wang, J.; Li, J.; Wang, F.; Zhao, L.; Wang, X.; Xiao, N.; Li, Y.; Wong, L.J.; et al. ADIPOR1 Is Mutated in Syndromic Retinitis Pigmentosa. Hum Mutat 2016, 37, 246-249, doi:10.1002/humu.22940.

20. Sundin, O.H.; Dharmaraj, S.; Bhutto, I.A.; Hasegawa, T.; McLeod, D.S.; Merges, C.A.; Silval, E.D.; Maumenee, I.H.; Lutty, G.A. Developmental basis of nanophthalmos: MFRP Is required for both prenatal ocular growth and postnatal emmetropization. Ophthalmic Genet 2008, 29, 1-9, doi:10.1080/13816810701651241.

21. Koli, S.; Labelle-Dumais, C.; Zhao, Y.; Paylakhi, S.; Nair, K.S. Identification of MFRP and the secreted serine proteases PRSS56 and ADAMTS19 as part of a molecular network involved in ocular growth regulation. PLoS Genet 2021, 17, e1009458, doi:10.1371/journal.pgen.1009458.

22. Kaarniranta, K.; Paananen, J.; Nevalainen, T.; Sorri, I.; Seitsonen, S.; Immonen, I.; Salminen, A.; Pulkkinen, L.; Uusitupa, M. Adiponectin receptor 1 gene (ADIPOR1) variant is associated with advanced age-related macular degeneration in Finnish population. Neurosci Lett 2012, 513, 233-237, doi:10.1016/j.neulet.2012.02.050.

23. Osada, H.; Toda, E.; Homma, K.; Guzman, N.A.; Nagai, N.; Ogawa, M.; Negishi, K.; Arita, M.; Tsubota, K.; Ozawa, Y. ADIPOR1 deficiency-induced suppression of retinal ELOVL2 and docosahexaenoic acid levels during photoreceptor degeneration and visual loss. Cell Death Dis 2021, 12, 458, doi:10.1038/s41419-021-03741-5.

24. Kautzmann, M.I.; Gordon, W.C.; Jun, B.; Do, K.V.; Matherne, B.J.; Fang, Z.; Bazan, N.G. Membrane-type frizzled-related protein regulates lipidome and transcription for photoreceptor function. FASEB $J$ 2020, 34, 912-929, doi:10.1096/fj.201902359R.

25. Sun, S.; Baryshnikova, A.; Brandt, N.; Gresham, D. Genetic interaction profiles of regulatory kinases differ between environmental conditions and cellular states. Mol Syst Biol 2020, 16, e9167, doi:10.15252/msb.20199167. 
26. Billmann, M.; Horn, T.; Fischer, B.; Sandmann, T.; Huber, W.; Boutros, M. A genetic interaction map of cell cycle regulators. Mol Biol Cell 2016, 27, 1397-1407, doi:10.1091/mbc.E15-07-0467.

27. Costanzo, M.; VanderSluis, B.; Koch, E.N.; Baryshnikova, A.; Pons, C.; Tan, G.; Wang, W.; Usaj, M.; Hanchard, J.; Lee, S.D.; et al. A global genetic interaction network maps a wiring diagram of cellular function. Science 2016, 353, doi:10.1126/science.aaf1420.

28. Boucher, B.; Jenna, S. Genetic interaction networks: better understand to better predict. Front Genet 2013, 4, 290, doi:10.3389/fgene.2013.00290.

29. Chakraborty, R.; Read, S.A.; Vincent, S.J. Understanding Myopia: Pathogenesis and Mechanisms. In Updates on Myopia, Ang, M., Wong, T.Y., Eds.; Springer: SIngapore, 2020; pp. 65-94.

30. Carter, G.W. Inferring network interactions within a cell. Brief Bioinform 2005, 6, 380-389, doi:10.1093/bib/6.4.380.

31. Papaleo, F.; Burdick, M.C.; Callicott, J.H.; Weinberger, D.R. Epistatic interaction between COMT and DTNBP1 modulates prefrontal function in mice and in humans. Mol Psychiatry 2014, 19, 311-316, doi:10.1038/mp.2013.133.

Fogerty, J.; Besharse, J.C. Subretinal infiltration of monocyte derived cells and complement misregulation in mice with AMD-like pathology. Adv Exp Med Biol 2014, 801, 355-363, doi:10.1007/978-1-4614-3209-8_45.

33. Gordon, W.C.; Bazan, N.G. Docosahexaenoic acid utilization during rod photoreceptor cell renewal. J Neurosci 1990, 10, 2190-2202.

34. Gordon, W.C.; Rodriguez de Turco, E.B.; Bazan, N.G. Retinal pigment epithelial cells play a central role in the conservation of docosahexaenoic acid by photoreceptor cells after shedding and phagocytosis. Curr Eye Res 1992, 11, 73-83, doi:10.3109/02713689209069169.

35. Gordon, W.C.; Bazan, N.G. Visualization of $[3 \mathrm{H}]$ docosahexaenoic acid trafficking through photoreceptors and retinal pigment epithelium by electron microscopic autoradiography. Investigative ophthalmology E visual science 1993, 34, $2402-2411$. Bazan, N.G. Overview of how N32 and N34 elovanoids sustain sight by protecting retinal pigment epithelial cells and photoreceptors. J Lipid Res 2021, 62, 100058, doi:10.1194/jlr.TR120001137.

37. Sluch, V.M.; Banks, A.; Li, H.; Crowley, M.A.; Davis, V.; Xiang, C.; Yang, J.; Demirs, J.T.; Vrouvlianis, J.; Leehy, B.; et al. ADIPOR1 is essential for vision and its RPE expression is lost in the Mfrp(rd6) mouse. Sci Rep 2018, 8, 14339, doi:10.1038/s41598-018-32579-9.

38. Almoallem, B.; Arno, G.; De Zaeytijd, J.; Verdin, H.; Balikova, I.; Casteels, I.; de Ravel, T.; Hull, S.; Suzani, M.; Destree, A.; et al. The majority of autosomal recessive nanophthalmos and posterior microphthalmia can be attributed to biallelic sequence and structural variants in MFRP and PRSS56. Sci Rep 2020, 10, 1289, doi:10.1038/s41598-019-57338-2.

39. Collery, R.F.; Volberding, P.J.; Bostrom, J.R.; Link, B.A.; Besharse, J.C. Loss of Zebrafish Mfrp Causes Nanophthalmia, Hyperopia, and Accumulation of Subretinal Macrophages. Investigative ophthalmology \& visual science 2016, 57, 6805-6814, doi:10.1167/iovs.16-19593.

40. Fuhrmann, S. Wnt signaling in eye organogenesis. Organogenesis 2008, 4, 60-67, doi:10.4161/org.4.2.5850.

41. Katoh, M. Molecular cloning and characterization of MFRP, a novel gene encoding a membrane-type Frizzled-related protein. Biochem Biophys Res Commun 2001, 282, 116-123, doi:10.1006/bbrc.2001.4551.

Krebs, M.P.; Xiao, M.; Sheppard, K.; Hicks, W.; Nishina, P.M. Bright-Field Imaging and Optical Coherence Tomography of the Mouse Posterior Eye. Methods Mol Biol 2016, 1438, 395-415, doi:10.1007/978-1-4939-3661-8_20.

43. Schindelin, J.; Arganda-Carreras, I.; Frise, E.; Kaynig, V.; Longair, M.; Pietzsch, T.; Preibisch, S.; Rueden, C.; Saalfeld, S.; Schmid, B.; et al. Fiji: an open-source platform for biological-image analysis. Nat Methods 2012, 9, 676-682, doi:10.1038/nmeth.2019.

44. Schaeffel, F.; Burkhardt, E.; Howland, H.C.; Williams, R.W. Measurement of refractive state and deprivation myopia in two strains of mice. Optom Vis Sci 2004, 81, 99-110, doi:10.1097/00006324-200402000-00008. 